\title{
The LOFAR Known Pulsar Data Pipeline
}

\author{
Anastasia Alexov*i \\ University of Amsterdam (UvA), Amsterdam, The Netherlands \\ E-mail: a.alexov@uva.nl \\ Jason W. T. Hessels \\ Netherlands Institute for Radio Astronomy (ASTRON), Dwingeloo, The Netherlands \\ University of Amsterdam (UvA), Amsterdam, The Netherlands \\ Jan David Mol \\ Netherlands Institute for Radio Astronomy (ASTRON), Dwingeloo, The Netherlands
}

\section{Ben Stappers}

The University of Manchester, Manchester, England

\section{Joeri van Leeuwen}

Netherlands Institute for Radio Astronomy (ASTRON), Dwingeloo, The Netherlands

University of Amsterdam (UvA), Amsterdam, The Netherlands

\begin{abstract}
Transient radio phenomena and pulsars are one of six LOFAR Key Science Projects (KSPs). As part of the Transients KSP, the Pulsar Working Group (PWG) has been developing the LOFAR Pulsar Data Pipelines to both study known pulsars as well as search for new ones. The pipelines are being developed for the Blue Gene/P (BG/P) supercomputer and a large Linux cluster in order to utilize enormous amounts of computational capabilities (50Tflops) to process data streams of up to 23TB/hour. The LOFAR pipeline output will be using the Hierarchical Data Format 5 (HDF5) to efficiently store large amounts of numerical data, and to manage complex data encompassing a variety of data types, across distributed storage and processing architectures. We present the LOFAR Known Pulsar Data Pipeline overview, the pulsar beam-formed data format, the status of the pipeline processing as well as our future plans for developing the LOFAR Pulsar Search Pipeline. These LOFAR pipelines and software tools are being developed as the next generation toolset for pulsar processing in Radio Astronomy.
\end{abstract}

ISKAF2010 Science Meeting - ISKAF2010

June 10-14, 2010

Assen, the Netherlands

\footnotetext{
*Speaker.

$\dagger$ Thank you to the LOFAR Pulsar Working Group and the LOFAR Transients Key Science Project
} 


\section{LOFAR Observing}

LOFAR operates in two frequency ranges: $10-80 \mathrm{MHz}$, using the Low Band Antennas (LBA), and $100-240 \mathrm{MHz}$, using the High Band Antennas (HBA). Close to 6000 antennas are distributed among 40 stations in the Netherlands, with several additional stations completed or planned throughout the rest of Europe. An observation can use subsets of any or all of the stations available. Radio interferometric imaging at $\sim 1$-second integration times is the main observing mode. However the high-time-resolution beam-formed observing modes will also be widely used for transient science. In this non-imaging mode, spatial resolution is traded for time resolution and time sampling can be as short as $5 \mu \mathrm{s}$. When multiple fields of view are synthesized simultaenously, the data rate can be as large as $23 \mathrm{~TB} / \mathrm{hr}$.

The Pulsar Working Group is in the process of creating automated pipeline processing software for studying and finding pulsars. This Pulsar Data Pipeline effort is the basis for other high-timingresolution pipelines using LOFAR to study, e.g., the Sun and planets.

\section{LOFAR Transient Science \& Requirements on Processing}

The processing of time-series (Beam-Formed) data has many scientific applications. The following areas of study set the requirements on processing for each of the envisioned sub-modes: (1) Known pulsars: channelization, Stokes parameters, dedispersion, folding, radio frequency interference (RFI) excision; (2) Pulsar/fast transient survey: channelization, dedispersion [1000s of trial dispersion measures (DMs)], RFI excision, searching; (3) Planets, Sun, flare stars: channelization, RFI excision, dynamic spectra.

Starting with LOFAR observations of known pulsars, the group has designed and implemented a pipeline for processing LOFAR time-series data, checking for pulsar detection, and analyzing the results [4]. The pipeline implements new and existing software tool sets for data processing, described in the sections below.

\section{Present LOFAR Beam-Formed (BF) Data Flow \& Processing}

The Beam-Formed data flow and processing are split into two broad, consecutive segments: "online" processing, which is done by a Blue Gene $\mathrm{P}(\mathrm{BG} / \mathrm{P})$ supercomputer to streaming data from the LOFAR stations, and "offline" processing which is further processing of these data on the LOFAR offline cluster. The online processing is chiefly responsible for combining data from multiple stations into one or multiple beams, as well as optionally producing correlations for imaging. The offline processing is more science-specific; it includes, for example dedispersion and folding of the data at a known pulsar period; it is even more flexible than the "online" processing system. Figure 1 shows the overall Beam-Formed data flow for a pulsar observation. The "online" and "offline" division is marked by the vertical red line.

Given that the processing of the raw station data is done entirely in software, the pipeline is quite flexible and extendable. Here we describe the current implementation. Many of the various online Pulsar Pipeline steps are common to other LOFAR observation modes. 


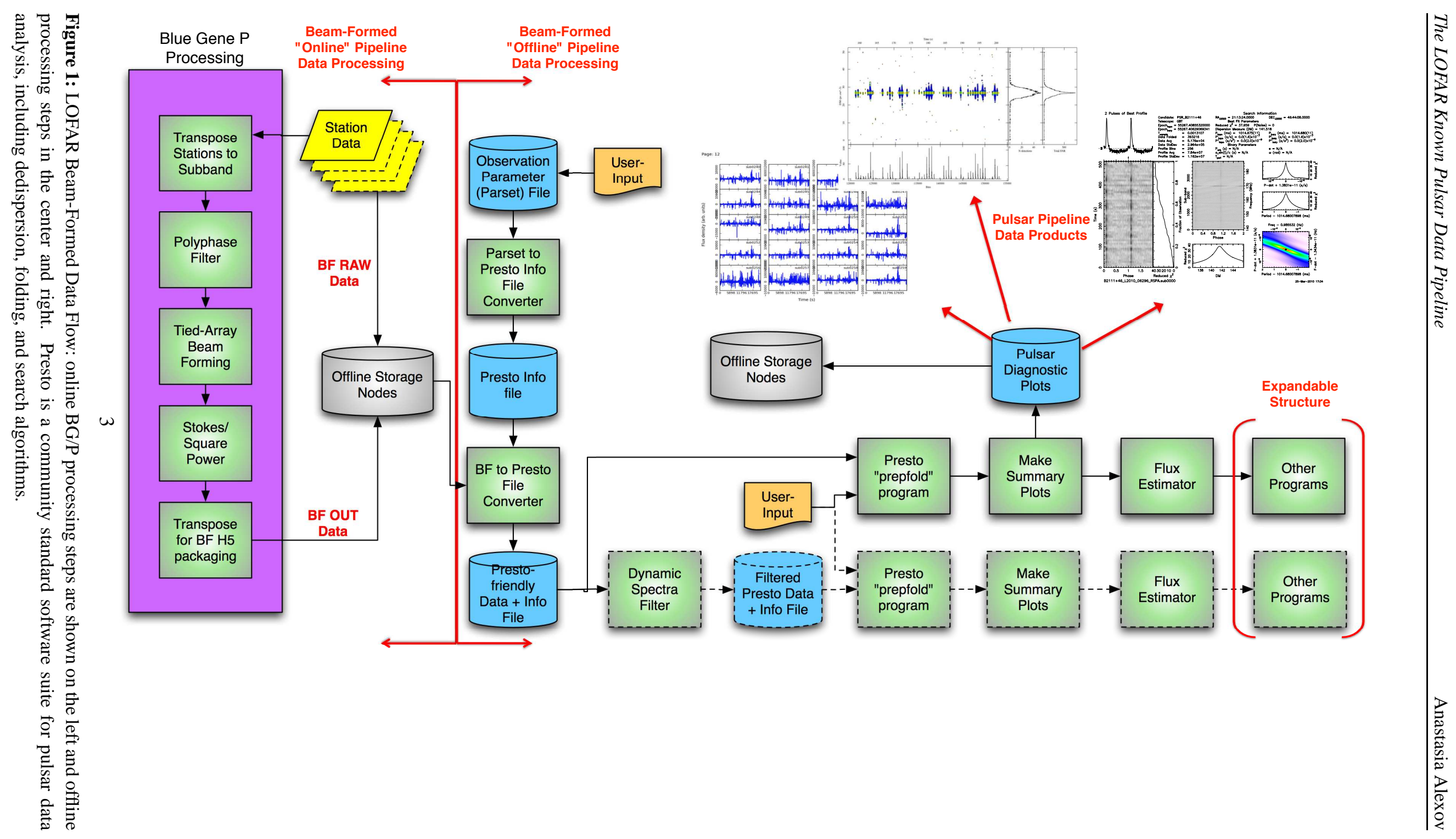




\subsection{Online: Tied-Array Beam Pipeline}

The station signals are fed to a Blue Gene P (BG/P) supercomputer in Groningen which can both combine the signals into coherent or incoherent array beams [6], or correlate them for imaging. These steps are on the left hand side of Figure 1, within the purple frame. In brief, the steps of the online Tied-Array Beam (TAB) Pipeline are: (1) First Transpose: for efficiency, data are transposed between stations and frequency subbands; (2) Second Polyphase Filter: an optional number of channels $(16$ - 256) is created within each $156 / 195-\mathrm{kHz}$ subband via a polyphase filter (PPF); (3) Beam-Forming: coherent and/or incoherent beam-forming between multiple stations (optional); (4) Stokes Parameters, Downsampling, and Re-binning: station signals from the $\mathrm{X}$ and $\mathrm{Y}$ polarizations of the antennas are used to derive the 4 Stokes parameters, I, Q, U, and V, or, to reduce the data rate by a factor of 4 , one can optionally output only Stokes I, the total intensity.

\subsection{Offline: Pipeline Processing}

With the station data now (optionally) combined into incoherent/coherent array beams, the goal of the offline processing is to apply further data-reduction. These steps are shown in the center and right hand side of Figure 1 as modules of the "offline" Known Pulsar Pipeline. In general, this pipeline performs RFI mitigation, dedispersion, and folding.

Offline processing happens on the LOFAR Offline Cluster, also in Groningen. The cluster is divided into subclusters, each of which contains $120 \mathrm{~TB}$ of disk storage and 9 compute nodes, with 2 quad-core processors each. In general, two subclusters are currently used for processing pulsar observations, which gives us over 200TB of disk space and 144 cores (split between 18 machines).

\subsubsection{Known Pulsar Pipeline}

For observations of known pulsars, the primary offline processing steps are dedispersion at the pulsar dispersion measure (DM) and folding using a pulsar ephemeris. These steps are automated in the Known Pulsar Pipeline that predominantly uses tools from the PRESTO ${ }^{1} \&$ TEMPO $^{2}$ software suites. Figure 1 (center and right) shows all the steps in the Offline Known Pulsar Pipeline; several steps are preparatory and data-format-related, followed by the use of science tools. Several pipeline processing modes are available: (1) Incoherent Stokes: station data are summed incoherently; (2) Coherent Stokes: station data are summed coherently; (3) Fly's Eye: station data are recorded separately for the purposes of system tests or to form a very large field of view.

RFI checking is also performed for long-term commissioning statistics, and for future RFImitigation. Pulsar diagnostic plots are produced (see top right of Figure 1) in order to quickly discern whether a pulsar was detected in the data. A typical 1-hour observation can be processed in 20 minutes using 8-cores on one machine, making it easy to reduce the data in realtime.

Since pipeline processing is modular, we can easily change the Known Pulsar Pipeline to use other pulsar tools if need be; we have the capability of using several file formats so that all existing pulsar tools can interface with the LOFAR data format. Several major upgrades (see Sections 3.2.3 and 3.2.4) are being worked on for the Known Pulsar Pipeline, which will also be applicable for other LOFAR pipelines, such as the Pulsar Search Pipeline.

\footnotetext{
${ }^{1}$ http://www.cv.nrao.edu//sransom/presto/

${ }^{2}$ http://www.atnf.csiro.au/research/pulsar/tempo/
} 


\subsubsection{Pulsar Search Pipeline}

We are in the process of testing a proto-type LOFAR Pulsar Search Pipeline, which uses several of the standard search tools from the PRESTO software suite. This proto-type pipeline is blindly detecting known pulsars in LOFAR data; it can manage RFI easily; and, it can run at close to real time if we use 9 compute nodes ( 72 cores in total) and search only for "slow" pulsars ( $P_{-}$spin $\gtrsim$ 50ms).

\subsubsection{Pipeline Framework}

Like other LOFAR processing pipelines, the Pulsar Pipelines are being built to run within a generic pipeline framework. This framework takes care of distributing the processing over the LOFAR offline cluster and its nodes, and provides appropriate logging and error checking[5]. The Pipeline Framework manages large parallel jobs in a more natural way, so that active observations and post-processing do not clash on the cluster. There is significant speedup by using the framework, which intelligently distributes the processing to all 9 machines within a subcluster, with 8 cores each. In the production system, the Pulsar Pipelines will start processing automatically after an observation.

\subsubsection{Data Format}

The data from the BG/P are currently written as almost header-less raw binary files and then converted to PRESTO-friendly format for pipeline processing. We can also convert data to SIGPROC $^{3}$ "filterbank" and "PSRFITS" [7] formats, thereby expanding the repertoire of pulsar tools we can use in the pipelines. The LOFAR Pulsar data format will soon be based on an implementation of the Hierarchical Data Format 5 (HDF5) ${ }^{4}$. The HDF5 file format was chosen because of its flexibility, its versatile data model, its ability to store very large, complex datasets spread over many separate physical devices. Anderson et al. (2010) discusses in depth the benefits of using HDF5 for LOFAR data storage, as well as the HDF5-tools which can be used for plotting and visualization of the data. The LOFAR Beam-Formed HDF5 data format is described in detail in the LOFAR Interface Control Document (ICD) \#3 [1].

\section{Software and Access}

As much as possible, the Pulsar Pipeline tries to use the well-tested, commonly used, open software packages available in the pulsar community (e.g. PRESTO, PSRCHIVE ${ }^{5}$ v13.0, SIGPROC v4.3, TEMPO v1.1 and Python bindings to PGPLOT, called ppgplot ${ }^{6}$ v1.1). These have been incorporated into the general LOFAR User Software (LUS ${ }^{7}$, which uses Cmake ${ }^{8}$, a crossplatform, open-source build system, to automate the installation of these packages on a variety of platforms (e.g. Ubuntu Linux and Mac OS X).

\footnotetext{
${ }^{3} \mathrm{http}: / /$ sigproc.sourceforge.net/

${ }^{4} \mathrm{HDF}$ home page: http://www.hdfgroup.org/

${ }^{5} \mathrm{http}: / /$ sourceforge.net/projects/psrchive/

${ }^{6}$ http://www.astro.rug.nl/ breddels/python/ppgplot/

${ }^{7}$ http://usg.lofar.org

${ }^{8}$ http://www.cmake.org/
} 
The current LOFAR Known Pulsar Pipeline uses PRESTO modules for folding data and viewing the results. PRESTO is a pulsar software tool suite that has been used within the pulsar community for almost 10 years. Several community-driven pulsar analysis tools complementary to PRESTO are being considered for LOFAR pulsar data pipeline processing - PSRCHIVE and SIGPROC. Both tool suites have had extensive use in the pulsar community and can be integrated as pipeline and analysis tools for LOFAR BF data. Using "off-the-shelf" software like PRESTO, SIGPROC and PSRCHIVE saves development time and also significant test and commissioning time, and, any software improvements to these tools will be fed back into the pulsar community.

\section{Beam-Formed Data Processing Enhancements}

In the coming year, a number of enhancements are anticipated for LOFAR Beam-Formed data processing: integrate the Known Pulsar Pipeline into the LOFAR Pipeline Framework to gain distributed processing, parallelization, logging and automated-kickoff after an observation; add coherent dedispersion to the Known Pulsar Pipeline; finalize the HDF5-writer to output BF data conforming to the LOFAR BF ICD; integrate pipeline tool suite with HDF5 data format; design Pulsar "searching" mode pipelines to reduce extreme data rates to few GB; automatically archive BF data products; implement "Piggyback" simultaneous observing with the LOFAR Million Source Shallow Survey (MSSS); add polarimetry and single pulse pulse data processing to the pipeline.

The LOFAR observatory is in the process of executing commissioning proposals, including pulsar observations. The current LOFAR Known Pulsar Pipeline has already been used to automatically process and detect more than 100 known pulsars [3]. The overall data-taking/processing/archiving system is being worked to accommodate pulsar and other LOFAR observations. The LOFAR Known and Search Pulsar Pipelines are anticipated to be fully automated within this system in the coming year, with many of the enhancement features listed above. This automation, parallel processing and distributed file-systems will help solve the enormous challenge of reducing $23 \mathrm{~TB} / \mathrm{hr}$ of data into several tens of GB of highly processed data: e.g., a pulsar "survey" observation is reduced to the parameters of the (new) candidate pulsars that were found!

\section{References}

[1] A. Alexov et al., LOFAR Data Format ICD: Beam-Formed Data, Document ID LOFAR-USG-ICD-003, Version 2.00.00, July 2010.

[2] K.R. Anderson et al., LOFAR and HDF5: Toward a New Radio Data Standard, in proceedings of The ISKAF2010 Science Meeting, 2010, POS (ISKAF2010) 062.

[3] J.W.T. Hessels, Early Pulsar Observations with LOFAR, in proceedings of The ISKAF2010 Science Meeting, 2010, POS ( ISKAF 2010 ) 025.

[4] B.W. Stappers et al., Pulsars \& LOFAR, University of Manchester, 2011 in prep.

[5] J. Swinbank, The LOFAR Transients Pipeline, in proceedings of The ISKAF2010 Science Meeting, 2010, POS (ISKAF 2010) 082.

[6] J. van Leeuwen and B.W. Stappers, Finding pulsars with LOFAR, A\&A, 509, 7, 2010.

[7] W. van Straten, R. N. Manchester, S. Johnston and J. Reynolds, Publ. Astron. Soc. Austral. 27 (2010) 104 [arXiv:0912.1662 [astro-ph.IM]]. 